\title{
Fuzzy Derivations BCC-Ideals on BCC-Algebras
}

\author{
Samy M. Mostafa, Mostafa A. Hassan \\ Department of mathematics, Faculty of Education, Ain Shams University Roxy, Cairo, Egypt \\ Email address: \\ samymostafa@yahoo.com (S. M. Mostafa),mostafamath1@yahoo.com (M. A. Hassan)
}

\section{To cite this article:}

Samy M. Mostafa, Mostafa A. Hassan. Fuzzy Derivations BCC-Ideals on BCC-Algebras. Pure and Applied Mathematics Journal. Vol. 4, No. 5, 2015, pp. 225-232. doi: 10.11648/j.pamj.20150405.14

\begin{abstract}
In the theory of rings, the properties of derivations are important. In [15], Jun and Xin applied the notion of derivations in ring and near-ring theory to BCI-algebras, and they also introduced a new concept called a regular derivation in BCI-algebras. They investigated some properties of its .In this manuscript, the concept of fuzzy left (right) derivations BCCideals in BCC-algebras is introduced and then investigate their basic properties. In connection with the notion of homomorphism, the authors study how the image and the pre-image of fuzzy left (right) derivations BCC-ideals under homomorphism of BCC-algebras become fuzzy left (right) derivations BCC-ideals. Furthermore, the Cartesian product of fuzzy left (right) derivations BCC-ideals in Cartesian product of BCC-algebras is introduced and investigated some related properties.
\end{abstract}

Keywords: BCC-Ideals, Fuzzy Left (Right)-Derivations, the Cartesian Product of Fuzzy Derivations

\section{Introduction}

In 1966 Iami and Iseki $[13,14]$ introduced the notion of BCK-algebras .Iseki $[11,12]$ introduced the notion of a BCIalgebra which is a generalization of BCK-algebra. Since then numerous mathematical papers have been written investigating the algebraic properties of the BCK / BCIalgebras and their relationship with other structures including lattices and Boolean algebras. A BCC-algebra is an important class of logical algebras introduced by Y. Komori [16] and was extensively investigated by many researcher's see $[1,3$, $4,5,6,7,8]$. The concept of fuzzy sets was introduced by Zadeh [21]. O. G. Xi [20] applied the concept of fuzzy set s to BCK-algebras. In the theory of rings, the properties of derivations are important. In [15], Jun and Xin applied the notion of derivations in ring and near-ring theory to BCIalgebras, and they also introduced a new concept called a regular derivation in BCI-algebras. They investigated some of its properties, defined a d-derivation ideal and gave conditions for an ideal to be d-derivation. Two years later, Hamza and Al-Shehri [9, 10] studied derivation in BCKalgebras, a left derivation in BCI-algebras and investigated a regular left derivation of BCI-algebras. C. Prabpayak, U. Leerawat [18] applied the notion of a regular derivation to BCC algebras and investigated some related properties.

In this paper, the authors consider the the concept of fuzzy left (right) derivations BCC-ideals in BCC-algebras and investigate some properties of it. Moreover, the concepts of the image and the pre-image of fuzzy left (right) derivations $\mathrm{BCC}$-ideals under homomorphism of BCC-algebras is given and studies some its properties. The Cartesian product of fuzzy left (right) derivations BCC-ideals in Cartesian product of BCC-algebras is introduced and investigated some related properties.

\section{Preliminaries}

In this section, we recall some basic definitions and results that are needed for our work.

Definition 2.1 [16] A BCC-algebra $(X, *, 0)$ is a nonempty set $\mathrm{X}$ with a constant 0 and a binary operation $*$ such that for all $x, y, z \in \mathrm{X}$ satisfying the following axioms:

$$
\begin{gathered}
(\mathrm{BCC}-1)((x * y) *(z * y)) *(x * z)=0 . \\
(\mathrm{BCC}-2) x * 0=x . \\
(\mathrm{BCC}-3) x * x=0 . \\
(\mathrm{BBC}-4) 0 * x=0 . \\
(\mathrm{BCC}-5) \quad x * y=y * x=0 \text { implies } x=y .
\end{gathered}
$$

Definition 2.2 [5] Let $(X, *, 0)$ be a BCC-algebra, we can define a binary relation $\leq$ on $X$ as, $x \leq y$ if and only if $x$ 
$* y=0$, this makes $(X, \leq)$ as a partially ordered set.

Proposition 2.3 [8] Let $(X, *, 0)$ be a BCC-algebra. Then the following hold $\forall x, y, z \in X$.

1. $(x * y) * x=0$.

2. $x \leq y$ implies $x * z \leq y * z$.

3. $x \leq y$ implies $z * y \leq z * x$.

4. $(x * y) *(z * y) \leq x * z$.

For elements $\mathrm{x}$ and $\mathrm{y}$ of a BCC-algebra $X=\left(X,{ }^{*}, 0\right)$ denote $x \wedge y=y^{*}\left(y^{*} x\right)$.

Lemma 2.4 [8] Let $\left(X,{ }^{*}, 0\right)$ be a BCC-algebra. Then the following hold $\forall x, y \in X$.

1. $0 \wedge x=0$

2. $x \wedge y \leq y$.

Example 2.5 [8] Let $X=\{0,1,2,3\}$ be a set in which the operation $*$ is defined as follows:

\begin{tabular}{lllll}
\hline$*$ & 0 & 1 & 2 & 3 \\
0 & 0 & 0 & 0 & 0 \\
1 & 1 & 0 & 1 & 0 \\
2 & 2 & 2 & 0 & 0 \\
3 & 3 & 3 & 1 & 0 \\
\hline
\end{tabular}

Then $\left(X,{ }^{*}, 0\right)$ is a BCC-algebra.

Definition $2.6[8]$ Let $(X, *, 0)$ be a BCC-algebra and $\mathrm{S}$ be a non-empty subset of $X$, then $\mathrm{S}$ is called subalgebra of $\mathrm{X}$ if $x * y \in S \quad \forall x, y \in S$.

Definition 2.7 [8] Let $\left(X,{ }^{*}, 0\right)$ be a BCC-algebra and A be a non-empty subset of $X$, then $\mathrm{A}$ is called ideal of $\mathrm{X}$ if it satisfied the following conditions:

1. $0 \in A$,

2. $x * y \in A, y \in A$ implies $x \in A \quad \forall x, y \in X$.

Definition 2.8 [8] Let $(X, *, 0)$ be a BCC-algebra and $A$ be a non-empty subset of $X$, then $A$ is called BCC- ideal of $X$ if it satisfied the following conditions:

1. $0 \in A$,

$$
(x * y) * z \in A, y \in A \text { implies } x * z \in A
$$

2. $\forall x, y, z \in X$.

Definition 2.9 [6] Let $\left(X,{ }^{*}, 0\right)$ be a BCC-algebra, a fuzzy set $\mu$ in $X$ is called a fuzzy subalgebra

$$
\text { if } \mu(x * y) \geq \min \{\mu(x), \mu(y)\} \quad \forall x, y \in X \text {. }
$$

Definition 2.10 [6] Let $(X, *, 0)$ be a BCC-algebra, a fuzzy set $\mu$ in $X$ is called a fuzzy BCC-ideal of $X$ if it satisfied the following conditions:

$$
\begin{aligned}
& \left(\mathrm{F}_{1}\right) \mu(0) \geq \mu(x), \\
& \left(\mathrm{F}_{2}\right) \mu(x * z) \geq \min \\
& \{\mu((x * y) * z), \mu(y)\} \quad \forall x, y, z \in X .
\end{aligned}
$$

Definition 2.11 [8] Let $\left(X,{ }^{*}, 0\right)$ is a BCC-algebra, $x, y \in X$,we denote $x \wedge y=y *(y * x)$.

Definition 2.12 [18] Let $\left(X,{ }^{*}, 0\right)$ be a BCC-algebra. A map $d: X \rightarrow X$ is called a left- right derivation (briefly (l,r)derivation) of $X$ if

$$
d(x * y)=(d(x) * y) \wedge(x * d(y)) \quad \forall x, y \in X .
$$

Similarly, a map $d: X \rightarrow X$ is called a right- left derivation (briefly $(r, l)$-derivation) of $X$ if

$$
\mathrm{d}(x * y)=(x * d(y)) \wedge(d(x) * y) \quad \forall x, y \in X .
$$

A map $d: X \rightarrow X$ is called a derivation of $X$ if $\mathrm{d}$ is both a $(l, r)$-derivation and a $(r, l)$-derivation of $X$.

Example 2.13 [18] Let $X=\{0,1,2,3\}$ be a BCC-algebra, in which the operation $*$ is defined as follows:

\begin{tabular}{l|llll}
\hline$*$ & 0 & 1 & 2 & 3 \\
0 & 0 & 0 & 0 & 0 \\
1 & 1 & 0 & 1 & 0 \\
2 & 2 & 2 & 0 & 0 \\
3 & 3 & 3 & 1 & 0 \\
\hline
\end{tabular}

Define a map $d: X \rightarrow X$ by

$$
d(x)=\left\{\begin{aligned}
0 & \text { if } x=0,1,3 \\
2 & \text { if } x=2 .
\end{aligned} \text {. Then it is clear that } d\right. \text { is a }
$$
derivation of $X$.

Example 2.14 Let $X=\{0,1,2,3\}$ be a BCC-algebra, in which the operation $*$ is defined as follows:

\begin{tabular}{l|llll}
\hline$*$ & 0 & 1 & 2 & 3 \\
0 & 0 & 0 & 0 & 0 \\
1 & 1 & 0 & 1 & 0 \\
2 & 2 & 2 & 0 & 0 \\
3 & 3 & 3 & 1 & 0 \\
\hline
\end{tabular}

Define a map $d: X \rightarrow X$ by

$$
d(x)= \begin{cases}x & \text { if } x=1,3 \\ 0 & \text { if } x=0,2\end{cases}
$$

Then $d$ is a $(r, l)$-derivation of $X$ but is not a $(l, r)$ derivation of $X$.

Definition 2.15 [18] Let be a BCC-algebra and $d: X$ $\rightarrow X$ be a map of a QS-algebra $X$, then $d$ is called regular if $d(0)=0$.

Lemma 2.16 [18] A derivation $d$ of BCC algebra $X$ is regular.

Proposition 2.17 [18] Let $\left(X,{ }^{*}, 0\right)$ be a BCC-algebra with partial order $\leq$, and let $d$ be a derivation of $X$. Then the following hold for all $x, y \in X$ :

1. $d(x) \leq x$,

2. $d(x * y) \leq d(x) * y$,

3. $d(x * y) \leq x * d(y)$,

4. $d(d(x)) \leq x$,

5. $d(x * d(x))=0$,

6. $d^{-1}(0)=\{x \in X: d(x)=0\}$ is a sub-algebra of $X$.

Definition 2.18 Let $X$ be a BCC-algebra and be a 
derivation of $X$.

Denote Fix $_{d}(X)=\{x \in X: d(x)=x\}$.

Proposition 2.19 [18] $X$ be a BCC-algebra and $d$ be a derivation of $X$. Then Fix $_{d}(X)$ is a subalgebra of $X$.

\section{Fuzzy Derivations BCC-Ideals on BCC-Algebras}

In this section, we will discuss and investigate a new notion is called fuzzy left (right) derivations BCC-ideals on BCC-algebras and study several basic properties which are related to fuzzy left (right) derivations BCC-ideals.

Definition 3.1 Let $\left(X,{ }^{*}, 0\right)$ be a BCC-algebra. and $d: X \rightarrow X$ be a self map. A non-empty subset $A$ of a BCCalgebra $X$ is called left derivations BCC-ideal of

If it satisfies the following conditions:

1. $0 \in A$,

2.

$$
d(x * y) * z \in A, d(y) \in A \text { implies } d(x * z) \in A
$$

$\forall x, y, z \in X$.

Definition 3.2 Let $(X, *, 0)$ be a BCC-algebra. and $d: X \rightarrow X$ be a self map. A non-empty subset $A$ of a BCCalgebra $X$ is called right derivations BCC-ideal of $X$

If it satisfies the following conditions:

1. $0 \in A$,

$(x * y) * d(z) \in A, d(y) \in A$ implies $d(x * z) \in A$

2. $\forall x, y, z \in X$.

Definition 3.3 Let $(X, *, 0)$ be a BCC-algebra. and $d: X \rightarrow X$ be a self map. A non-empty subset $A$ of a BCCalgebra $X$ is called derivations BCC-ideal of $X$

If it satisfies the following conditions:

1. $0 \in A$,

2.

$$
\begin{aligned}
& d((x * y) * z) \in A, d(y) \in A \text { implies } d(x * z) \in A \\
& \forall x, y, z \in X .
\end{aligned}
$$

Definition 3.4 Let $\left(X,{ }^{*}, 0\right)$ be a BCC-algebra. and $d: X \rightarrow X$ be a self map. A fuzzy set $\mu: X \rightarrow[0,1]$ in $X$ is called a fuzzy left derivations BCC-ideal (briefly $(F, l)$ derivation) of $X$ if it satisfies the following conditions:

$$
\begin{gathered}
\left(\mathrm{F}_{1}\right) \mu(0) \geq \mu(x) \quad \forall x \in X, \\
\left(\mathrm{FL}_{2}\right) \quad \begin{array}{l}
\mu(d(x * z)) \geq \min \{\mu(d(x) \\
\forall x, y, z \in X .
\end{array}
\end{gathered}
$$

Definition 3.5 Let $\left(X,{ }^{*}, 0\right)$ be a BCC-algebra. and $d: X \rightarrow X$ be a self map. A fuzzy set $\mu: X \rightarrow[0,1]$ in $X$ is called a fuzzy right derivations BCC-ideal (briefly $(F, r)$ derivation) of $X$ if it satisfies the following conditions:

$$
\left(\mathrm{F}_{1}\right) \mu(0) \geq \mu(x) \quad \forall x \in X,
$$

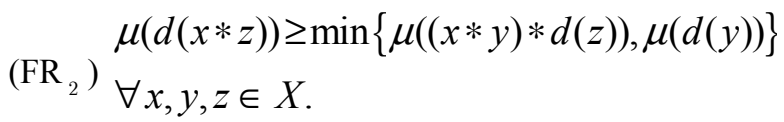

Definition 3.6 Let $\left(X,{ }^{*}, 0\right)$ be a BCC-algebra. and $d: X \rightarrow X$ be a self map.

A fuzzy set $\mu: X \rightarrow[0,1]$ in $X$ is called a fuzzy derivations BCC-ideal of $X$ if it satisfies the following conditions:

$$
\begin{aligned}
& \left(\mathrm{F}_{1}\right) \mu(0) \geq \mu(d(x)) \quad \forall x \in X, \\
& \left(\mathrm{~F}_{2}\right) \begin{array}{l}
\mu(d(x * z)) \geq \min \{\mu(d((x * y) * z)), \mu(d(y))\} \\
\forall x, y, z \in X .
\end{array}
\end{aligned}
$$

Remark 3.7

1. If $d$ is fixed, the definitions (3.1., 3.2., 3.3.) gives the definition BCC-ideal.

2. If $d$ is fixed, the definitions (3.4., 3.5., 3.6.) gives the definition fuzzy BCC-ideal.

Example 3.8 Let $X=\{0,1,2,3\}$ be a BCC-algebra, in which the operation $*$ is defined as follows:

\begin{tabular}{l|llll}
\hline$*$ & 0 & 1 & 2 & 3 \\
0 & 0 & 0 & 0 & 0 \\
1 & 1 & 0 & 1 & 0 \\
2 & 2 & 2 & 0 & 0 \\
3 & 3 & 3 & 1 & 0 \\
\hline
\end{tabular}

Define a map $d: X \rightarrow X$ by

$$
d(x)= \begin{cases}0 & \text { if } x=0,1,3 \\ 2 & \text { if } x=2\end{cases}
$$

Define a fuzzy set $\mu: d(x) \rightarrow[0,1]$, by $\mu(d(0))=t_{0}$, $\mu(d(1))=t_{1}, \quad \mu(d(2))=\mu(d(3))=t_{2}, \quad$ where $t_{0}, \quad t_{1}$, $t_{2} \in[0,1]$ with $t_{0}>t_{1}>t_{2}$. Routine calculations give that $\mu$ is not fuzzy left (right)-derivations BCC-ideal of BCCalgebra.

Example 3.9 Let $X=\{0,1,2,3,4,5\}$ be a BCC-algebra, in which the operation $*$ is defined as follows:

\begin{tabular}{lllllll}
\hline$*$ & 0 & 1 & 2 & 3 & 4 & 5 \\
0 & 0 & 0 & 0 & 0 & 0 & 0 \\
1 & 1 & 0 & 0 & 0 & 0 & 1 \\
2 & 2 & 2 & 0 & 0 & 1 & 1 \\
3 & 3 & 2 & 1 & 0 & 1 & 1 \\
4 & 4 & 4 & 4 & 4 & 0 & 1 \\
5 & 5 & 5 & 5 & 5 & 5 & 0 \\
\hline
\end{tabular}

Define a map $d: X \rightarrow X$ by

$$
d(x)=\left\{\begin{array}{cl}
0 & \text { if } x=0,1,2,3,4 \\
5 & \text { if } x=5
\end{array}\right.
$$

Define a fuzzy set $\mu: d(x) \rightarrow[0,1]$, by $\mu(d(0))=t_{0}$, $\mu(d(1))=t_{1}, \quad \mu(d(2))=\mu(d(3))=t_{2}$, $\mu(d(4))=\mu(d(5))=t_{2}$, where $t_{0}, \quad t_{1}, \quad t_{2} \in[0,1]$ with 
$t_{0}>t_{1}>t_{2}$. Routine calculations give that $\mu$ is fuzzy left (right)-derivations BCC-ideal of BCC-algebra.

Theorem 3.10 Let $\mu$ be a fuzzy left derivations BCC-ideal of BCC-algebra $X$.

1. If $x \leq d(y) \quad$, then $\quad \mu(d(x)) \geq \mu(d(y))$

2. If $x * y \leq d(x)$, then $\mu(d(x * y)) \geq \mu(d(x))$

3 . If $(x * y) *(z * y) \leq d(x * z)$, then

$\mu(d((x * y) *(z * y))) \geq \mu(d(x * z))$

4. If $\mu(d(x * y))=\mu(d(0))$, then $\mu(d(x)) \geq \mu(d(y))$.

Pr oof. 1. Let $x \leq d(y)$ and since $\overbrace{d(y) \leq y}^{\text {from } \operatorname{Pr} o 2.16 .1}$,

hence $x \leq y$ i.e. $x * y=0$, then

$$
\begin{aligned}
& \mu(d(x))=\mu(d(x * 0)) \geq \overbrace{\min \{\mu(d(x * y) * 0), \mu(d(y))\}}^{\text {from Def } 3.4 .\left(F L_{2}\right)} \\
& =\min \{\mu(d(x * y)), \mu(d(y))\} \\
& =\min \{\mu(d(0)), \mu(d(y))\} \\
& =\min \{\mu(0), \mu(d(y))\}=\mu(d(y)) .
\end{aligned}
$$

2. Let $x * y \leq d(x)$, then by Theorem 3.10.1, we get $\mu(d(x * y)) \geq \mu(d(x))$.

3. Let $(x * y) *(z * y) \leq d(x * z)$,

then by theorem 3.10.1, we get

$\mu(d((x * y) *(z * y))) \geq \mu(d(x * z))$.

4. Let $\mu(d(x * y))=\mu(d(0))$, then

$\mu(d(x))=\mu(d(x * 0))$

$\geq \overbrace{\min \{\mu(d(x * y) * 0), \mu(d(y))\}}^{\text {from Def 3.4. }\left(F L_{2}\right)}$

$=\min \{\mu(d(x * y)), \mu(d(y))\}$

$=\min \{\mu(d(0)), \mu(d(y))\}=\min \{\mu(0), \mu(d(y))\}=\mu(d(y))$.

Proposition 3.11 The intersection of any set of fuzzy left derivations BCC-ideals of BCC-algebra $X$ is also fuzzy left derivations BCC-ideal.

Proof. Let $\left\{\mu_{i}\right\}$ be a family of fuzzy left derivations BCC-ideals of BCC-algebra $X$,

then $\forall x, y, z \in X$,

$$
\begin{aligned}
& \left(\bigcap \mu_{i}\right)(0)=\inf \left(\mu_{i}(0)\right) \geq \inf \left(\mu_{i}(d(x))\right)=\left(\bigcap \mu_{i}\right)(d(x)) \text { and } \\
& \left(\bigcap \mu_{i}\right)(d(x * z))=\inf \left(\mu_{i}(d(x * z))\right. \\
& \geq \inf \left(\min \left\{\mu_{i}(d(x * y) * z), \mu_{i}(d(y))\right\}\right. \\
& =\min \left\{\inf \left(\mu_{i}(d(x * y) * z)\right), \inf \left(\mu_{i}(d(y))\right)\right\} \\
& =\min \left\{\left(\bigcap \mu_{i}\right)(d(x * y) * z),\left(\bigcap \mu_{i}\right)(d(y))\right\} . \quad \text { Lemma }
\end{aligned}
$$

3.12 The intersection of any set of fuzzy right derivations BCC-ideals of BCC-algebra $X$ is also fuzzy right derivations BCC-ideal.

\section{Pr oof. Clear}

Theorem 3.13 Let $\mu$ be a fuzzy set in $X$, then $\mu$ is a fuzzy left derivations BCC-ideal of $X$ if and only if it satisfies : $\forall$ $\alpha \in[0,1]), U(\mu, \alpha) \neq \varphi$ implies $\mathrm{U}(\mu, \alpha)$ is BCC-ideal of $X \ldots(\mathrm{A})$, where $\mathrm{U}(\mu, \alpha)=\{\mathrm{x} \in \mathrm{X} / \mu(\mathrm{d}(\mathrm{x})) \geq \alpha\}$.

Proof. Assume that $\mu$ is a fuzzy left derivations BCCideal of $X$, let $\alpha \in[0,1]$ be such that $\mathrm{U}(\mu, \alpha) \neq \phi$ and $x, y \in X$ such that $x \in \mathrm{U}(\mu, \alpha)$, then $\mu(d(x)) \geq \alpha$ and so by $\left(\mathrm{FL}_{2}\right)$,

$$
\begin{aligned}
& \mu(d(0))=\mu(d(0 * y)) \geq \min \{\mu(d(0 * x) * y), \mu(d(x))\} \\
& =\min \{\mu(d(0) * y), \mu(d(x))\} \\
& =\min \{\mu(0 * y), \mu(d(x))\}=\min \{\mu(0), \mu(d(x))\}=\alpha, \\
& \text { hence } 0 \in \mathrm{U}(\mu, \alpha) .
\end{aligned}
$$

Let $d(x * y) * z \in \mathrm{U}(\mu, \alpha)$ and $d(y) \in(\mu, \alpha)$,

it follows from $\left(\mathrm{FL}_{2}\right)$ that

$$
\mu(d(x * z)) \geq \min \{\mu(d(x * y) * z), \mu(d(y))\}=\alpha,
$$

so that $x * z \in \mathrm{U}(\mu, \alpha)$. Hence $\mathrm{U}(\mu, \alpha)$ is

BCC-ideal of $X$.

Conversely, suppose that $\mu$ satisfies (A), let $x, y, z \in X$ be such that

$$
\begin{aligned}
& \qquad \mu(d(x * z))<\min \{\mu(d(x * y) * z), \mu(d(y))\}, \quad \text { taking } \\
& \beta_{0}=1 / 2\{\mu(d(x * z))+\min \{\mu(d(x * y) * z), \mu(d(y))\}\}, \\
& \text { we have } \beta_{0} \in[0,1] \text { and } \\
& \qquad \mu(d(x * z))<\beta_{0}<\min \{\mu(d(x * y) * z), \mu(d(y))\},
\end{aligned}
$$

it follows that $d(x * y) * z \in \mathrm{U}\left(\mu, \beta_{0}\right)$ and $\mathrm{U}\left(\mu, \beta_{0}\right)$, this is a contradiction and therefore $\mu$ is a fuzzy left derivations BCC-ideal of $X$.

Theorem 3.14 Let $\mu$ be a fuzzy set in $X$, then $\mu$ is a fuzzy right derivations BCC-ideal of $X$ if and only if it satisfies : $\forall \alpha \in[0,1]), \mathrm{U}(\mu, \alpha) \neq \varphi$ implies $\mathrm{U}(\mu, \alpha)$ is BCC-ideal of $X \ldots(\mathrm{A})$, where $\mathrm{U}(\mu, \alpha)=\{\mathrm{x} \in \mathrm{X} / \mu(\mathrm{d}(\mathrm{x})) \geq \alpha\}$.

Proof. Clear

Definition 3.15 Let $\mu$ be a fuzzy derivations BCC-ideal of BCC-algebra $X$, the BCC-ideals $\mu_{i}, t \in[0,1]$ are called level BCC-ideal of $\mu$.

\section{Image (Pre-image) of Fuzzy Derivations BCC-Ideals Under Homomorphism}

In this section, we introduce the concepts of the image and the pre-image of fuzzy left and right derivations BCC-ideals in BCC-algebras under homomorphism of BCC-algebras

Definition 4.1 Let $f$ be a mapping from the set $X$ to a set 
$Y$. If $\mu$ is a fuzzy subset of $X$, then the fuzzy subset $\beta$ of $Y$ is defined by

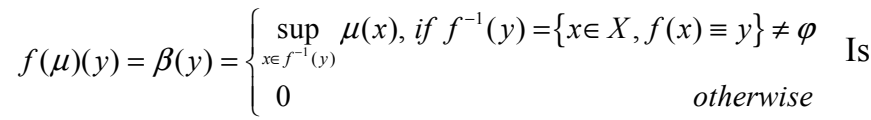
said to be the image of $\mu$ under $f$

Similarly if $\beta$ is a fuzzy subset of $Y$, then the fuzzy subset $\mu=\beta$ of in $X$ (i.e. the fuzzy subset is defined by $\mu(x)=\beta(f(x)) \quad \forall x \in X)$ is called the preimage of $\beta$ under $f$.

Theorem 4.2 An onto homomorphic preimage of a fuzzy right derivations $\mathrm{BCC}$-ideal is also a fuzzy right derivations BCC-ideal under homomorphism of BCC-algebras

Proof. Let $f: X \rightarrow X^{\prime}$ be an onto homomorphism of BCC-algebras, $\beta$ a fuzzy right derivations BCC-ideal of $X^{\prime}$ and $\mu$ the preimage of $\beta$ under $f$, then $\beta(f(d(x)))=\mu(d(x)), \quad \forall x \in X . \quad$ Let $\quad x \in X, \quad$ we have $\mu(d(0))=\beta(f(d(0))) \geq \beta(f(d(x)))=\mu(d(x))$.

Now let $x, y, z \in X$, then

$$
\mu(d(x * z))=\beta(f(d(x * z)))
$$$$
\geq \min \left\{\beta\left(\left(f(x) *^{\prime} f(y)\right) *^{\prime} f(d(z))\right), \beta(f(d(y)))\right\}
$$$$
=\min \{\beta((x * y) * f(d(z))), \beta(f(d(y)))\}
$$$$
=\min \{\mu((x * y) * d(z)), \mu(d(y))\} \text {. }
$$

The proof is completed.

Theorem 4.3 An onto homomorphic preimage of a fuzzy left derivations BCC-ideal is also a fuzzy left derivations BCC-ideal.

\section{Pr oof. Clear}

Definition 4.4 [2] A fuzzy subset $\mu$ of $X$ has sup property if for any subset $T$ of $X$,

there exist $t_{0} \in T$ such that, $\mu\left(t_{0}\right)=\sup _{t \in T} \mu(t)$.

Theorem 4.5 Let $f: X \rightarrow Y$ be a homomorphism between BCC-algebras $X$ and $Y$. For every fuzzy left derivations BCC-ideal $\mu$ in $X, f(\mu)$ is a fuzzy left derivations BCC-ideal of $Y$.

Pr oof. By definition

$$
\beta\left(d\left(y^{\prime}\right)\right)=f(\mu)\left(d\left(y^{\prime}\right)\right)=\sup _{d(x) \in f^{-1}\left(d\left(y^{\prime}\right)\right)} \mu(d(x))
$$

$\forall y^{\prime} \in Y$ and $\sup \varphi=0$. We have to prove that $\beta\left(d\left(x^{\prime} * z^{\prime}\right)\right) \geq \min \left\{\beta\left(d\left(x^{\prime} * y^{\prime}\right) * z^{\prime}\right), \beta\left(d\left(y^{\prime}\right)\right)\right\}$, $\forall x^{\prime}, y^{\prime}, z^{\prime} \in Y$.

Let $f: X \rightarrow Y$ be an onto a homomorphism of BCCalgebras, $\mu$ a fuzzy left derivations BCC-ideal of $X$ with sup property and $\beta$ the image of $\mu$ under $f$, since $\mu$ is a fuzzy left derivations BCC-ideal of $X$, we have $\mu(\mathrm{d}(0)) \geq \mu(\mathrm{d}(\mathrm{x}))$

$\forall \mathrm{x} \in X$. Note that $0 \in f^{-1}\left(0^{\prime}\right)$, where $0,0^{\prime}$ are the zero of $X$ and $Y$ respectively. Thus, $\beta\left(d\left(0^{\prime}\right)\right)=\sup _{d(t) \in f^{-1}\left(d\left(0^{\prime}\right)\right)} \mu(d(t))=\mu(d(0))=\mu(0) \geq \mu(d(x))$,

$\forall x \in X$, which implies that

$\beta\left(d\left(0^{\prime}\right)\right) \geq \sup _{d(t) \in f^{-1}\left(d\left(x^{\prime}\right)\right)} \mu(d(t))=\beta\left(d\left(x^{\prime}\right)\right), \quad \forall \quad x^{\prime} \in Y . \forall$

$x^{\prime}, y^{\prime}, z^{\prime} \in Y$, let

$d\left(x_{0}\right) \in f^{-1}\left(d\left(x^{\prime}\right)\right)$,

$d\left(y_{0}\right) \in f^{-1}\left(d\left(y^{\prime}\right)\right)$

$d\left(z_{0}\right) \in f^{-1}\left(d\left(z^{\prime}\right)\right)$

be such that

$$
\begin{aligned}
& \mu\left(d\left(x_{0} * z_{0}\right)\right)=\sup _{d(t) \in f^{-1}\left(d\left(x^{\prime} * z^{\prime}\right)\right)} \mu(d(t)), \mu\left(y_{0}\right) \\
& =\sup _{d(t) \in f^{-1}\left(d\left(y^{\prime}\right)\right)} \mu(d(t)) \\
& \mu\left(d\left(x_{0} * y_{0}\right) * z_{0}\right)=\beta\left\{f\left(d\left(x_{0} * y_{0}\right) * z_{0}\right)\right\} \\
& =\beta\left(d\left(x^{\prime} * y^{\prime}\right) * z^{\prime}\right) \quad \mu\left(d\left(x_{0} * y_{0}\right) * z_{0}\right) \\
& =\sup _{\left(d\left(x_{0} * y_{0}\right) * z_{0}\right) \in f^{-1}\left(d\left(x^{\prime} * y^{\prime}\right) * z^{\prime \prime}\right)} \mu(d(t)) . \\
& =\sup _{d(t) \in f^{-1}\left(d\left(x^{\prime} * y^{\prime}\right) * z^{\prime}\right)} \mu(d(t)
\end{aligned}
$$

Then

$$
\begin{aligned}
& \quad \beta\left(d\left(x^{\prime} * z^{\prime}\right)\right)=\sup _{d(t) \in f^{-1}\left(d\left(x^{\prime} * z^{\prime}\right)\right)} \mu(d(t))=\mu\left(d\left(x_{0} * z_{0}\right)\right) \\
& \geq \min \left\{\mu\left(d\left(x_{0} * y_{0}\right) * z_{0}\right), \mu\left(d\left(y_{0}\right)\right)\right\}= \\
& \min \left\{\sup _{d(t) \in f^{-1}\left(d\left(x^{\prime} * y^{\prime}\right) * z^{\prime}\right)} \mu(d(t)), \quad \sup _{d(t) \in f^{-1}\left(d\left(y^{\prime}\right)\right)} \mu(d(t))\right\}= \\
& \min \left\{\beta\left(d\left(x^{\prime} * y^{\prime}\right) * z^{\prime}\right), \quad \beta\left(d\left(y^{\prime}\right)\right)\right\} .
\end{aligned}
$$

Hence $\beta$ is a fuzzy left derivations BCC-ideal of $Y$.

Theorem 4.6 Let $f: X \rightarrow Y$ be a homomorphism between BCC-algebras $X$ and $Y$. For every fuzzy right derivations BCC-ideal $\mu$ in $X, f(\mu)$ is a fuzzy right derivations BCC-ideal of $Y$.

\section{Proof. Clear}

\section{Cartesian Product of Fuzzy Left Derivations BCC-ideals}

Definition 5.1 [2] A fuzzy $\mu$ is called a fuzzy relation on any set $S$, if $\mu$ is a fuzzy subset $\mu: S \times S \rightarrow[0,1]$.

Definition 5.2 [2] If $\mu$ is a fuzzy relation on a set $S$ and is a fuzzy subset of $S$, then $\mu$ is a fuzzy relation on $\beta$ if $\mu(x, y) \leq \min \{\beta(x), \beta(y)\}, \quad \forall x, y \in S$.

Definition 5.3 [2] Let $\mu$ and $\beta$ be a fuzzy subset of a set $S$, the Cartesian product of $\mu$ and $\beta$ is defined by $(\mu \times \beta)(x, y)=\min \{\mu(x), \beta(y)\}, \quad \forall x, y \in S$.

Lemma 5.4 [2] Let $\mu$ and $\beta$ be a fuzzy subset of a set $S$, then 
(i) $\mu \times \beta$ is a fuzzy relation on $S$.

$(i i)(\mu \times \beta)_{t}=\mu_{t} \times \beta_{t} \quad \forall t \in[0,1]$.

Definition 5.5 If $\mu$ is a fuzzy derivations relation on a set $S$ and $\beta$ is a fuzzy derivations subset of $S$, then $\mu$ is a fuzzy derivations relation on $\beta$ if

$$
\mu(d(x, y)) \leq \min \{\beta(d(x)), \beta(d(y))\}, \quad \forall x, y \in S .
$$

Definition 5.6 Let $\mu$ and $\beta$ be a fuzzy derivations subset of a set $S$, the Cartesian product of $\mu$ and $\beta$ is defined by $(\mu \times \beta)(d(x, y))=\min \{\mu(d(x)), \beta(d(y))\}, \quad \forall x, y \in S$.

Definition 5.7 If $\beta$ is a fuzzy derivations subset of a set $S$, the strongest fuzzy relation on $S$, that is a fuzzy derivations relation on $\beta$ is $\mu_{\beta}$ given by

$$
\mu_{\beta}(d(x, y))=\min \{\beta(d(x)), \beta(d(y))\}, \quad \forall x, y \in S .
$$

Lemma 5.8 [2] For a given fuzzy derivations subset of a set $S$, let $\mu_{\beta}$ be the strongest fuzzy derivations relation on $S$, then for $t \in[0,1]$, we have $\left(\mu_{\beta}\right)_{t}=\beta_{t} \times \beta_{t}$.

Proposition 5.9 For a given fuzzy derivations subset $\beta$ of BCC-algebra $X$, let $\mu_{\beta}$ be the strongest fuzzy derivations relation on $X$. If $\mu_{\beta}$ is a fuzzy derivations BCC-ideal of $X \times X$, then $\beta(d(x)) \leq \beta(d(0))=\beta(0) \quad \forall x \in X$.

Pr oof. Since $\mu_{\beta}$ is a fuzzy derivations BCC-ideal of $X \times X$, it follows from $\left(\mathrm{F}_{1}\right)$ that

$\mu_{\beta}(x, x)=\min \{\beta(d(x)), \beta(d(x))\} \leq \beta(d(0,0))$

$=\min \{\beta(d(0)), \beta(d(0))\}$

where $(0,0) \in X \times X$, then $\beta(d(x)) \leq \beta(d(0))=\beta(0)$.

Remark 5.10 Let $X$ and $Y$ be BCC-algebras, we define $*$ on $X \times Y$ by

$(x, y) *(u, v)=(x * u, y * v) \quad \forall(x, y),(u, v) \in X \times Y$, then clearly $(X \times Y, *,(0,0))$ is a BCC-algebra.

Theorem 5.11 Let $\mu$ and $\beta$ be a fuzzy derivations BCCideals of BCC-algebra $X$, then $\mu \times \beta$ is a fuzzy derivations BCC-ideal of $X \times X$.

Proof. 1.

$(\mu \times \beta)(d(0,0))=\min \{\mu(d(0)), \beta(d(0))\}=\min \{\mu(0), \beta(0)\}$

$\geq \min \{\mu(d(x)), \beta(d(x))\}=(\mu \times \beta)(d(x, y))$

$\forall(x, y) \in X \times X$.

2. Let $\left(x_{1}, x_{2}\right),\left(y_{1}, y_{2}\right),\left(z_{1}, z_{2}\right) \in X \times X$, then

$(\mu \times \beta)\left(d\left(x_{1} * z_{1}, x_{2} * z_{2}\right)\right)=\min \left\{\mu\left(d\left(x_{1}, z_{1}\right)\right), \beta\left(d\left(x_{2}, z_{2}\right)\right)\right\}$

$\geq \min \left\{\begin{array}{l}\min \left\{\mu\left(d\left(\left(x_{1} * y_{1}\right) * z_{1}\right)\right), \mu\left(d\left(y_{1}\right)\right)\right\}, \\ \min \left\{\beta\left(d\left(\left(x_{2} * y_{2}\right) * z_{2}\right)\right), \beta\left(d\left(y_{2}\right)\right)\right\}\end{array}\right\}$ $=\min \left\{\begin{array}{l}\min \left\{\mu\left(d\left(\left(x_{1} * y_{1}\right) * z_{1}\right)\right), \beta\left(d\left(\left(x_{2} * y_{2}\right) * z_{2}\right)\right)\right\}, \\ \min \left\{\mu\left(d\left(y_{1}\right)\right), \beta\left(d\left(y_{2}\right)\right)\right\}\end{array}\right\}$
$=\min \left\{\begin{array}{l}(\mu \times \beta)\left(\left(d\left(\left(x_{1} * y_{1}\right) * z_{1}\right)\right),\left(d\left(\left(x_{2} * y_{2}\right) * z_{2}\right)\right)\right), \\ (\mu \times \beta)\left(d\left(y_{1}\right), d\left(y_{2}\right)\right)\end{array}\right\}$.

Hence $\mu \times \beta$ is a fuzzy derivations BCC-ideal of $X \times X$.

Analogous to theorem 2.2[17], we have a similar result for fuzzy derivations BCC-ideal, which can be proved in similar manner, we state the result without proof.

Theorem 5.12 Let $\mu$ and $\beta$ be a fuzzy derivations subset of BCC-algebra $X$, Such that $\mu \times \beta$ is a fuzzy derivations BCC-ideal of $X \times X$, then

(i) either $\mu(d(x)) \leq \mu(d(0))$ or $\beta(d(x)) \leq \beta(d(0)) \quad \forall x \in X$,

(ii) if $\mu(d(x)) \leq \mu(d(0)) \quad \forall x \in X$, then either $\mu(d(x)) \leq \beta(d(0))$ or $\beta(d(x)) \leq \beta(d(0))$,

(iii) if $\beta(d(x)) \leq \beta(d(0)) \quad \forall x \in X$, then either $\mu(d(x)) \leq \mu(d(0))$ or $\beta(d(x)) \leq \mu(d(0))$,

(iv) either $\mu$ or $\beta$ is a fuzzy derivations BCC-ideal of $X$.

Theorem 5.13 Let $\beta$ be a fuzzy derivations subset of BCC-algebra $X$ and let $\mu_{\beta}$ be the strongest fuzzy derivations relation on then $\beta$ is a fuzzy derivations BCC-ideal of $X$ if and only if $\mu_{\beta}$ is a fuzzy derivations BCC-ideal of $X \times X$.

$\operatorname{Pr}$ oof. Let $\beta$ be a fuzzy derivations BCC-ideal of $X, 1$. From $\left(\mathrm{F}_{1}\right)$, we get

$$
\begin{aligned}
& \mu_{\beta}(0,0)=\min \{\beta(d(0)), \beta(d(0))\}=\min \{\beta(0), \beta(0)\} \geq \\
& \min \{\beta(d(x)), \beta(d(y))\}=\mu_{\beta}(d(x), d(y))
\end{aligned}
$$

2. $\forall\left(x_{1}, x_{2}\right),\left(y_{1}, y_{2}\right),\left(z_{1}, z_{2}\right) \in X \times X$, we have from $\left(\mathrm{F}_{2}\right)$ $\mu_{\beta}\left(d\left(x_{1} * z_{1}\right), d\left(x_{2} * z_{2}\right)\right)=\min \left\{\beta\left(d\left(x_{1} * z_{1}\right)\right), \beta\left(d\left(x_{2} * z_{2}\right)\right)\right\}$ $\geq \min \left\{\begin{array}{l}\min \left\{\beta\left(d\left(\left(x_{1} * y_{1}\right) * z_{1}\right)\right), \beta\left(d\left(y_{1}\right)\right)\right\}, \\ \min \left\{\beta\left(d\left(\left(x_{2} * y_{2}\right) * z_{2}\right)\right), \beta\left(d\left(y_{2}\right)\right)\right\}\end{array}\right\}$ $=\min \left\{\begin{array}{l}\min \left\{\beta\left(d\left(\left(x_{1} * y_{1}\right) * z_{1}\right)\right), \beta\left(d\left(\left(x_{2} * y_{2}\right) * z_{2}\right)\right)\right\}, \\ \min \left\{\beta\left(d\left(y_{1}\right)\right), \beta\left(d\left(y_{2}\right)\right)\right\}\end{array}\right\}$ $=\min \left\{\begin{array}{l}\mu_{\beta}\left(\left(d\left(\left(x_{1} * y_{1}\right) * z_{1}\right)\right),\left(d\left(\left(x_{2} * y_{2}\right) * z_{2}\right)\right)\right), \\ \mu_{\beta}\left(d\left(y_{1}\right), d\left(y_{2}\right)\right)\end{array}\right\}$.

Hence $\mu_{\beta}$ is a fuzzy derivations BCC-ideal of $X \times X$.

Conversely, let $\mu_{\beta}$ be a fuzzy derivations BCC-ideal of $X \times X$,

1. $\forall(x, y) \in X \times X$, we have $\min \{\beta(0), \beta(0)\}=\mu_{\beta}(x, y)=\min \{\beta(x), \beta(y)\}$.

It follows that $\beta(0) \geq \beta(x) \quad \forall x \in X$, which prove $\left(\mathrm{F}_{1}\right)$. 
2. Let $\left(x_{1}, x_{2}\right),\left(y_{1}, y_{2}\right),\left(z_{1}, z_{2}\right) \in X \times X$, then

$$
\min \left\{\beta\left(d\left(x_{1} * z_{1}\right)\right), \beta\left(d\left(x_{2} * z_{2}\right)\right)\right\}
$$

$\mu_{\beta}\left(d\left(x_{1} * z_{1}\right), d\left(x_{2} * z_{2}\right)\right)$

$\geq \min \left\{\mu_{\beta}\left(d\left(\left(x_{1}, x_{2}\right) *\left(y_{1}, y_{2}\right)\right) *\left(z_{1}, z_{2}\right)\right), \mu_{\beta}\left(d\left(y_{1}\right), d\left(y_{2}\right)\right)\right\}$

$=\min \left\{\begin{array}{l}\mu_{\beta}\left(d\left(\left(x_{1} * y_{1}\right) * z_{1}\right), d\left(\left(x_{2} * y_{2}\right) * z_{2}\right)\right), \\ \mu_{\beta}\left(d\left(y_{1}\right), d\left(y_{2}\right)\right)\end{array}\right\}$

$=\min \left\{\begin{array}{l}\min \left\{\beta\left(d\left(\left(x_{1} * y_{1}\right) * z_{1}\right)\right), \beta\left(d\left(\left(x_{2} * y_{2}\right) * z_{2}\right)\right)\right\}, \\ \min \left\{\beta\left(d\left(y_{1}\right)\right), \beta\left(d\left(y_{2}\right)\right)\right\}\end{array}\right\}$

$=\min \left\{\begin{array}{l}\min \left\{\beta\left(d\left(\left(x_{1} * y_{1}\right) * z_{1}\right)\right), \beta\left(d\left(y_{1}\right)\right)\right\}, \\ \min \left\{\beta\left(d\left(x_{2} * y_{2}\right) * z_{2}\right), \beta\left(d\left(y_{2}\right)\right)\right\}\end{array}\right\}$

In particular, if we take $x_{2}=y_{2}=z_{2}=0$, then

$\beta\left(d\left(x_{1} * z_{1}\right)\right) \geq \min \left\{\beta\left(d\left(x_{1} * y_{1}\right) * z_{1}\right), \beta\left(d\left(y_{1}\right)\right)\right\} \quad$. This

prove $\left(\mathrm{F}_{2}\right)$

Hence $\beta$ be a fuzzy derivations BCC-ideal of $X$.

\section{Conclusion}

Derivation is a very interesting and important area of research in the theory of algebraic structures in mathematics. In the present paper, the notion of fuzzy left and right derivations BCC-ideal in BCC-algebra are introduced and investigated the useful properties of fuzzy left and right derivations $\mathrm{BCC}$-ideals in $\mathrm{BCC}$-algebras.

In our opinion, these definitions and main results can be similarly extended to some other algebraic systems such as BCI-algebra, BCH-algebra, Hilbert algebra, BF-algebra, Jalgebra, WS-algebra, CI-algebra, SU-algebra, BCL-algebra, BP-algebra, Coxeter algebra, BO-algebra, PU- algebras and so forth.

The main purpose of our future work is to investigate:

(1) The interval value, bipolar and intuitionist fuzzy left and right derivations $\mathrm{BCC}$-ideal in BCC-algebra.

(2) To consider the cubic structure left and right derivations BCC- ideal in BCC-algebra.

We hope the fuzzy left and right derivations BCC-ideals in $\mathrm{BCC}$-algebras, have applications in different branches of theoretical physics and computer science.

\section{Algorithm for BCC-algebras}

Input ( $X$ : set, $*$ : binary operation)

Output (" $X$ is a BCC-algebra or not")

Begin

If $X=\varphi$ then go to (1.);

End If

If $0 \notin X$ then go to (1.);

End If

Stop: $=$ false;

$i:=1$;
While $i \leq|X|$ and not (Stop) do

If $x_{i} * x_{i} \neq 0$ then

Stop: $=$ true;

End If

$j:=1$

$k:=1$

While $j, k \leq|X|$ and not (Stop) do

If $\left.\left(\left(x_{i} * y_{j}\right) *\left(z_{k} * y_{j}\right)\right) *\left(x_{i} * z_{k}\right)\right) \neq 0$ then

Stop: $=$ true;

End If

End While

End While

If Stop then

Output (" $X$ is not a BCC-algebra")

Else

Output (" $X$ is a BCC-algebra")

End If

End.

\section{References}

[1] S. M. Bawazeer, N. O. Alshehri, and Rawia Saleh Babusail, "Generalized Derivations of BCC-Algebras," International Journal of Mathematics and Mathematical Sciences, volume 2013, Article ID 451212, 4 pages.

[2] P. Bhattacharye and N. P. Mukheriee, Fuzzy relations and fuzzy group inform, sci, 36(1985), 267-282.

[3] W. A. Dudek, Y. B. Jun, Zoran Stojakovic, "On fuzzy ideals in BCC-algebras," Fuzzy Sets and Systems 123 (2001) 251-258.

[4] W. A. Dudek . The number of subalgebras of finite BCCalgebras, Bull. Inst. Math. Acad. Sinica, 20 (1992), 129-136.

[5] W. A. Dudek., On proper BCC-algebras, Bull. Inst. Math. Acad. Sinica, 20 (1992), 137-150.

[6] W. A. Dudek. and Y. B Jun, , Fuzzy BCC-ideals in BCCalgebras, Math. Montisnigri, 10 (1999), 21-30.

[7] W. A. Dudek. and Y. B Jun and C. Z .Stojakovi, On fuzzy ideals in BCCalgebras, Fuzzy Sets and Systems, 123 (2001), 251-258.

[8] W. A. Dudek, and X.H Zhang, On ideals and congruences in BCCalgebras, Czechoslovak Math. J., 48 (123) (1998), 21-29.

[9] A. S. A Hamza and N. O. Al-Shehri. 2006. Some results on derivations of BCI-algebras. Coden Jnsmac 46: 13-19.

[10] A. S. A Hamza and N. O. Al-Shehri. 2007. On left derivations of BCI-algebras. Soochow Journal of Mathematics 33(3): 435-444.

[11] Y. Huang, BCI-algebra, Science Press, Beijing, 2006.

[12] K. Is'eki, "On BCI-algebras," Mathematics Seminar Notes, vol. 8, no. 1, pp. 125-130, 1980.

[13] K. Is'eki and S. Tanaka, "An introduction to the theory of BCKalgebras," Mathematica Japonica, vol. 23, no. 1, pp. 1-26, 1978.

[14] K. Is'eki and S. Tanaka, "Ideal theory of BCK-algebras," Mathematica Japonica, vol. 21, no. 4, pp. 351-366, 1976. 
[15] Y. B. Jun, X. L. Xin. 2004. On derivations ofBCI-algebras. Information Sciences 159:167-176.

[16] Y. Komori, The class of BCC-algebras is not a variety, Math. Japonica, 29 (1984), 391-394.

[17] D. S. Malik and J. N. Mordeson, Fuzzy relation on rings and groups, Fuzzy Sets and Systems 43 (1991) 117-123.

[18] C. Prabpayak, Um Leerawat,On Derivations of BCC-algebras. Kasetsart J. (Nat. Sci.) 43: 398 - 401 (2009).
[19] A. Wro_nski, BCK-algebras do not form a variety, Math. Japonica, 28 (1983), 211-213.

[20] O. G. Xi, Fuzzy BCK-algebras, Math. Japon. 36 (1991), 935 942.

[21] L. A. Zadeh, Fuzzy sets, Information and Control, 8 (1965), 338-353. 